\title{
On Spectrum of the Linearized 3D Euler Equation
}

\author{
Roman Shvydkoy and Misha Vishik \\ Communicated by Charles Li, received February 26, 2004.
}

\begin{abstract}
We investigate essential spectrum of the Euler equation linearized about an arbitrary smooth steady flow in dimension 3 . It is proved that for every Lyapunov-Oseledets exponent $\mu$ of the associated bicharacteristicamplitude system, the circle of radius $e^{\mu t}$ has a common point with the spectrum. If, in addition, $\mu$ is attained on an aperiodic point, then the spectrum contains the entire circle.
\end{abstract}

\section{Contents}

1. Introduction

2. Hyperbolic Semigroups 53

3. Aperiodic Case 55

4. Action of $L$

5. Functions with Zero Lyapunov Index 59

References

\section{Introduction}

Studying stability of equilibrium solutions to a nonlinear PDE, it is crucial to understand asymptotic behavior of the corresponding linearized equation. For the system of Euler equations governing the motion of an ideal incompressible fluid,

$$
\begin{gathered}
u_{t}=-(u, \nabla) u-\nabla p, \\
\operatorname{div} u=0
\end{gathered}
$$

this has been the subject of a classical theory originated in works of Rayleigh, Kelvin, Love and others $[\mathbf{3}, \mathbf{6}, \mathbf{1 3}, \mathbf{1 7}]$. In its early stage a prime emphasis was given to the search of unstable eigenvalues (i.e. eigenvalues with positive real part)

1991 Mathematics Subject Classification. Primary 76E09; Secondary 34D09.

Key words and phrases. Linearized Euler equation, essential spectrum, Lyapunov-Oseledets exponents.

The work of the second author was partially supported by NSF grant DMS 9876947. 
for particular examples of equilibria such as parallel shear flow. We refer to recent works $[\mathbf{1}, \mathbf{9}, \mathbf{1 0}, \mathbf{1 5}]$ for modern developments in this direction. The results we will present concern examination of so-called essential part of the spectrum, which may as well become a source of (linear) instability.

So, we consider a steady solution to (1.1), (1.2), denoted $u(x)$, on the torus $\mathbb{T}^{n}$. Our choice of boundary conditions is motivated by purely technical reasons. Other types such as the free space or bounded domain should not present major difficulties. We also assume sufficient smoothness for $u(x)$ where necessary, and this will be our only restriction on $u$.

Neglecting non-linear terms, the evolution equation for small perturbations of $u$ is written as follows

$$
\begin{gathered}
v_{t}=-(u, \nabla) v-(v, \nabla) u-\nabla p \\
\operatorname{div} v=0
\end{gathered}
$$

The nature of the spectrum of the Euler operator defined from (1.3) by

$$
L v=-(u, \nabla) v-(v, \nabla) u-\nabla p
$$

and the corresponding evolution semigroup $G_{t}=e^{t L}, t \geq 0$, is sensitive to the choice of norm in which instability is measured. For our discussion, we take the physically reasonable energy norm. So, the semigroup acts on the space of divergence-free square-integrable vector fields on the torus, written $L_{\text {div }}^{2}\left(T^{n}\right), n=2,3$.

For a general bounded operator $G$ on a Banach space, its spectrum $\sigma(G)$ splits into union of discrete and essential parts. Following Browder [2] we call $\lambda \in \sigma(G)$ a point of discrete spectrum, $\sigma_{\text {disc }}(G)$, if (the third condition given in $[\mathbf{2}]$ is redundant for bounded operators)

(1d) $\lambda$ is an isolated point in $\sigma(G)$,

(2d) the corresponding spectral subspace, determined by the image of the Riesz projection

$$
P=\frac{1}{2 \pi i} \int_{|z-\lambda|<\delta} \frac{d z}{z-G},
$$

is finite dimensional.

The essential spectrum, denoted by $\sigma_{\text {ess }}(G)$, is defined as $\sigma(G) \backslash \sigma_{\text {disc }}(G)$. Similar classification can be given for any unbounded closed operator.

A complete description for the essential spectrum of the linear Euler equation in dimension two has been recently given in $[\mathbf{1 9}]$. To state the result, let $\varphi_{t}: \mathbb{T}^{n} \rightarrow \mathbb{T}^{n}$ be the integral measure-preserving flow of the steady field $u(x)$. The maximal Lyapunov exponent of the Jacobi matrix $\partial \varphi_{t}(x)=\left(\frac{\partial \varphi_{t}^{i}(x)}{\partial x_{j}}\right)_{i, j=1}^{n}$ is defined by the formula

$$
\Lambda=\lim _{t \rightarrow \infty} \frac{1}{t} \log \max _{x \in \mathbb{T}^{n}}\left\|\partial \varphi_{t}(x)\right\| .
$$

Then, for $n=2$, the following identities hold:

$$
\begin{aligned}
\sigma_{\mathrm{ess}}\left(G_{t}\right) & =\left\{e^{-t \Lambda} \leq|z| \leq e^{t \Lambda}\right\}, \\
\sigma_{\mathrm{ess}}(L) & =\{|\operatorname{Re} z| \leq \Lambda\}
\end{aligned}
$$

Consequently, we obtain the spectral mapping theorem:

$$
\sigma\left(G_{t}\right) \backslash\{0\}=e^{t \sigma(L)} .
$$


In dimension three, however, description of essential spectrum becomes increasingly difficult. The problem arises from the presence of additional vortex stretching term. In the vorticity formulation of (1.3) it adds a generically bounded perturbation to the advective derivative, whereas in two dimensions it adds only compact one. To bypass this difficulty a new method was deployed in $[\mathbf{1 1}, \mathbf{2 1}]$ based on WKB-type expansion of localized highly oscillating perturbations. More specifically, suppose initially

$$
v(x, t=0)=b_{0}\left(x, \xi_{0}\right) e^{i \xi_{0} \cdot x / \delta},
$$

where $\delta$ is small, the amplitude $b_{0}$ is localized around $x_{0}$ and orthogonal to the wave vector $\xi_{0}$ to insure incompressibility. The corresponding solution can be expanded into a formal series in powers of $\delta$,

$$
v(x, t)=b\left(x, \xi_{0}, t\right) e^{i S\left(x, \xi_{0}, t\right) / \delta}+\ldots
$$

Substituting this into (1.3) and disregarding the lower powers of $\delta$ we obtain the following system of ODE written in Lagrangian coordinates

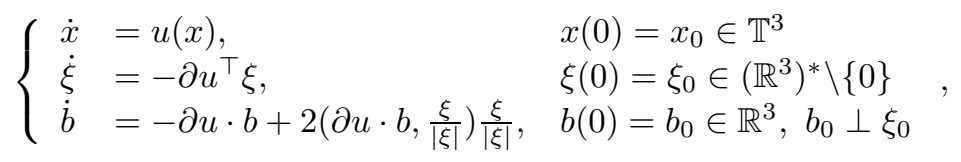

where $\xi=\nabla S$. It is referred to as the bicharacteristic-amplitude system associated with $u(x)$ (see [8] for details). Its dynamical content is the following.

The first two equations define a flow on $\mathbb{K}=\mathbb{T}^{3} \times \mathbb{P R}^{2}$ given by

$$
(x, \xi) \rightarrow\left(\varphi_{t}(x), \frac{\partial \varphi_{t}^{-\top}(x) \xi}{\left|\partial \varphi_{t}^{-\top}(x) \xi\right|}\right),
$$

where '- $T$ ' means 'inverse transpose', and $\mathbb{P R}^{2}$ denotes the two dimensional projective space. The corresponding mapping

$$
\left(x_{0}, \xi_{0}, b_{0}\right) \rightarrow b\left(t, x_{0}, \xi_{0}, b_{0}\right)
$$

generated by solutions of the third equation in (1.5) defines a linear cocycle over this flow (see $[4,14])$ :

$$
B_{t}\left(x_{0}, \xi_{0}\right) b_{0}=b\left(t, x_{0}, \xi_{0}, b_{0}\right) .
$$

Since the b-equation is homogenous in $\xi$ and smooth in both variables, so is the cocycle. Furthermore, since $\mathbb{K}$ is compact, the classical Oseledets' Multiplicative Ergodic Theorem $[\mathbf{4}, \mathbf{1 6}]$ applies to yield a set of points $\mu$, called Lyapunov-Oseledets exponents, for each of which there is a $\left(x_{0}, \xi_{0}\right) \in \mathbb{K}$ and $b_{0} \in \mathbb{R}^{3}$ such that

$$
\mu=\lim _{t \rightarrow \infty} \frac{1}{t} \log \left|B_{t}\left(x_{0}, \xi_{0}\right) b_{0}\right| \text {. }
$$

It can be shown that the maximal exponent exists and is equal to

$$
\mu_{\max }=\lim _{t \rightarrow \infty} \frac{1}{t} \log \max _{(x, \xi) \in \mathbb{K}}\left\|B_{t}(x, \xi)\right\|
$$

(see [4]). This dynamical quantity serves as a replacement for the upper Lyapunov exponent of the basic flow $\varphi_{t}$ in three dimensions. In fact, the following formula for the essential spectral radius was proved in $[\mathbf{2 0}]$

$$
r_{\mathrm{ess}}\left(G_{t}\right)=e^{t \mu_{\max }},
$$


(see also [18]). This formula was effectively used in a number of examples to detect instabilities caused by essential spectrum, $[\mathbf{9}, \mathbf{1 1}]$. For us it gives a piece of information about the structure of the spectrum, namely $\sigma_{\text {ess }}\left(G_{t}\right) \cap\left\{|z|=e^{t \mu_{\max }}\right\} \neq$ $\emptyset$. Our first result is a direct generalization of this fact.

Theorem 1.1. Suppose $u(x)$ is a smooth equilibrium solution of the Euler equation (1.1) on $\mathbb{T}^{3}$. Let $\mu$ be any Lyapunov-Oseledets exponent of the associated system (1.5). Then for all $t>0$,

$$
\sigma_{\text {ess }}\left(G_{t}\right) \cap\left\{|z|=e^{t \mu}\right\} \neq \emptyset .
$$

We prove this theorem in two steps. First, we establish a general sufficient condition for (1.7) based on existence of a weakly null sequence of unit vectors $\left\{x_{n}\right\}_{n=1}^{\infty}$ such that for each $t>0,\left\|G_{t} x_{n}\right\|$ tends to $f(t)$, which as a function of $t$ has Lyapunov index $\mu$. Then, we adopt a construction from $[\mathbf{1 8}, \mathbf{2 0}]$ and produce such a sequence for the Euler semigroup.

By analogy with the two dimensional case, one may conjecture that

$$
\sigma_{\text {ess }}\left(G_{t}\right)=\left\{e^{-t \mu_{\max }} \leq|z| \leq e^{t \mu_{\max }}\right\} .
$$

This amounts to proving two facts: $\sigma_{\text {ess }}\left(G_{t}\right)$ has no circular gaps, and $\sigma_{\text {ess }}\left(G_{t}\right)$ is rotationally invariant. It was shown in $[\mathbf{1 8}, \mathbf{2 0}]$ (see Section 2) that, modulo a compact perturbation, the Euler semigroup is representable in a form very similar to that of the evolution Mather semigroup associated with the b-cocycle $B_{t}(x, \xi)$. Due to de la Llave's theorem [5], a Mather semigroup has no circular spectral gaps when defined on a space of divergence-free vector fields. Even though it remains open whether such a property is inherited by the Euler semigroup, this at least suggests the validity of the first fact.

As to the second, unfortunately, all known mechanisms producing rotational invariance of the spectrum require presence of long periodic or non-periodic orbits in the underlying flow. In two dimensions, this is automatically provided by existence of a hyperbolic point whenever $\Lambda>0$. Fully three dimensional equilibria, on the other hand, may exhibit a much more complicated topology in which even periodic flows can produce positive Lyapunov exponents.

This examination shows that more truthful, although wide open to date, statement would be the annular hull version of (1.8),

$$
\bigcup_{0 \leq \theta \leq 2 \pi} e^{i \theta} \sigma_{\mathrm{ess}}\left(G_{t}\right)=\left\{e^{-t \mu_{\max }} \leq|z| \leq e^{t \mu_{\max }}\right\} .
$$

The second part of our paper addresses a special case, in which more refined description of the spectrum can be given.

A point $x_{0} \in \mathbb{T}^{3}$ is called aperiodic if its every neighborhood is crossed by an orbit of $\left\{\varphi_{t}\right\}$ with arbitrary large (possibly infinite) period. A Lyapunov-Oseledets exponent is called aperiodic if the point $x_{0}$ in formula (1.6) can be chosen aperiodic. As discussed previously, availability of long orbits is suggestive to rotational invariance. Indeed, the following result is true.

TheOREM 1.2. Suppose $u(x)$ is a smooth equilibrium solution of the Euler equation (1.1) on $\mathbb{T}^{3}$. Let $\mu$ be an aperiodic Lyapunov-Oseledets exponent of (1.5). Then for all $t>0$,

$$
\left\{|z|=e^{t \mu}\right\} \subset \sigma_{\text {ess }}\left(G_{t}\right) .
$$


We leave the essential spectrum of the generator for future study. Let us remark that the highly oscillating functions used in the method present the main obstacle in proving similar results for $L$. It is possible to suppress the oscillations in the situation when given Lyapunov exponent is attained for $\xi_{0}$ and $x_{0}$ satisfying $\xi_{0} \perp u\left(x_{0}\right)$. This special case is treated in article [14], where, in particular, the authors prove that the exponential growth type of the semigroup and the spectral bound of the generator are equal.

\section{Hyperbolic Semigroups}

We start with a few general remarks on $C_{0}$-semigroups. Our immediate goal is to find a simple sufficient condition for non-hyperbolicity, which will eventually lead us to the proof of Theorem 1.1.

Let us recall that a $C_{0}$-semigroup $\left\{G_{t}\right\}_{t \geq 0}$ of bounded operators on a Banach space $X$ is called hyperbolic provided $X$ can be split into a direct sum of two subspaces $X_{s}$ and $X_{u}$ invariant with respect to the semigroup, and such that for some $\varepsilon>0$ and $M>0$ one has

(1h) $\left\|G_{t} x\right\| \leq M e^{-\varepsilon t}\|x\|$, for all $x \in X_{s}$ and $t>0$;

(2h) $\left\|G_{t} x\right\| \geq M^{-1} e^{\varepsilon t}\|x\|$, for all $x \in X_{u}$ and $t>0$.

The semigroup $\left\{G_{t}\right\}_{t \geq 0}$ is hyperbolic if and only if $\sigma\left(G_{t}\right) \cap \mathbb{T}=\emptyset$ for all - or, equivalently, for some $t>0$ (see [7]). If this is the case, the operators

$$
\begin{aligned}
& P_{s}=\frac{1}{2 \pi i} \int_{\mathbb{T}} \frac{d z}{z-G_{t}} \\
& P_{u}=I-P_{s}
\end{aligned}
$$

define projection maps onto the corresponding subspaces $X_{s}, X_{u}$, and

$$
\begin{aligned}
& \sigma\left(\left.G_{t}\right|_{X_{s}}\right)=\sigma\left(G_{t}\right) \cap\left\{|z| \leq e^{-\varepsilon t}\right\} \\
& \sigma\left(\left.G_{t}\right|_{X_{u}}\right)=\sigma\left(G_{t}\right) \cap\left\{|z| \geq e^{\varepsilon t}\right\}
\end{aligned}
$$

A simple rescaling argument shows that for every $\lambda \in \mathbb{R}$, the new semigroup operator $e^{\lambda t} G_{t}$ has no spectrum inside the annulus $\left\{e^{(\lambda-\varepsilon) t} \leq|z| \leq e^{(\lambda+\varepsilon) t}\right\}$.

Analogously, we say that the semigroup $\left\{G_{t}\right\}_{t \geq 0}$ is essentially hyperbolic if $\sigma_{\text {ess }}\left(G_{t}\right) \cap \mathbb{T}=\emptyset$ for all $t>0$. Equivalently, there are invariant subspaces $X_{s}, X_{c}, X_{u}$ which split $X$ into a direct sum, such that conditions (1h), (2h) are satisfied for $X_{s}, X_{u}$, and $X_{c}$ is finite dimensional.

In this terminology, Theorem 1.1 simply states that $\left\{e^{-\mu t} G_{t}\right\}_{t \geq 0}$ is not essentially hyperbolic for every Lyapunov-Oseledets exponent $\mu$ of the bicharacteristic system (1.5). So, to prove the theorem we first establish a simple sufficient condition for non-essential hyperbolicity of a general $C_{0}$-semigroup. Then based on construction from $[\mathbf{1 8}, \mathbf{2 0}]$ we verify this condition for the Euler semigroup.

Proposition 2.1. Let $\left\{G_{t}\right\}_{t \geq 0}$ be a $C_{0}$-semigroup on a Banach space $X$. Suppose there is a sequence of unit vectors $\left\{x_{n}\right\}_{n=1}^{\infty} \subset X$ such that

(1p) $\lim _{n \rightarrow \infty}\left\|G_{t} x_{n}\right\|=f(t)$ for all $t>0$,

(2p) $\lim _{t \rightarrow \infty} \frac{1}{t} \log f(t)=\mu$.

Then $\left\{e^{-\mu t} G_{t}\right\}_{t \geq 0}$ is not hyperbolic. If, in addition, $x_{n} \rightarrow 0$ weakly in $X$, then $\left\{e^{-\mu t} G_{t}\right\}_{t \geq 0}$ is not essentially hyperbolic, i.e.

$$
\sigma_{\mathrm{ess}}\left(G_{t}\right) \cap e^{\mu t} \mathbb{T} \neq \emptyset .
$$


Proof. After rescaling we can assume that $\mu=0$. Also, assume on the contrary that $\left\{G_{t}\right\}_{t \geq 0}$ is hyperbolic. Then there exist invariant subspaces $X_{s}$ and $X_{u}$ which satisfy conditions (1h), (2h) with some $\varepsilon>0$. Let $x_{n}=x_{n}^{s}+x_{n}^{u}$ where $x_{n}^{s} \in X_{s}$ and $x_{n}^{u} \in X_{u}$. We show that $\left\|x_{n}^{u}\right\| \rightarrow 0$.

Indeed, fix an arbitrary $\delta>0$. According to (2p) there is a $t>0$ such that $0<f(t)<e^{\varepsilon t / 2}$. Furthermore, by (1p), for sufficiently large $n \in \mathbb{N}$ we find $\left\|G_{t} x_{n}\right\| \leq 2 f(t)$. This implies, via (1h) and (2h), that for some positive $c$,

$$
2 e^{\varepsilon t / 2}>\left\|G_{t} x_{n}\right\| \geq c\left\|G_{t} x_{n}^{s}\right\|+c\left\|G_{t} x_{n}^{u}\right\| \geq c M^{-1} e^{\varepsilon t}\left\|x_{n}^{u}\right\| .
$$

Thus, $\left\|x_{n}^{u}\right\| \leq 2 c^{-1} M e^{-\varepsilon t / 2}$. Choosing $t$ so that $2 c^{-1} M e^{-\varepsilon t / 2}<\delta$ we obtain the desired result.

Since $\left\|x_{n}^{u}\right\| \rightarrow 0$ as $n \rightarrow \infty$, condition (1p) can now be replaced by $\lim _{n \rightarrow \infty}\left\|G_{t} x_{n}^{s}\right\|=$ $f(t)$ for each $t>0$. Then in view of (1h), $f(t) \leq c e^{-\varepsilon t}$ for all $t>0$, which contra$\operatorname{dicts}(2 \mathrm{p})$.

Let us assume that $x_{n} \rightarrow 0$ weakly, and $\sigma\left(G_{t}\right) \cap \mathbb{T} \subset \sigma_{\text {disc }}\left(G_{t}\right)$. Then the spectral subspace $X_{c}$ corresponding to the part of the spectrum lying on $\mathbb{T}$ is finite dimensional. The other two subspaces $X_{s}$ and $X_{u}$ satisfy (1h) and (2h) as before. Also, $X=X_{s} \oplus X_{c} \oplus X_{u}$. We decompose each $x_{n}$ into the sum $x_{n}^{u}+x_{n}^{c}+x_{n}^{s}$. If $\operatorname{dim} X_{c}=N$, we let

$$
x_{n}^{c}=\sum_{j=1}^{N} y_{j}^{*}\left(x_{n}\right) y_{j}
$$

for some basis $\left\{y_{j}\right\}_{j=1}^{N}$ in $X_{c}$ and $\left\{y_{j}\right\}_{j=1}^{N} \subset X^{*}$. Since $x_{n} \rightarrow 0$ weakly, $y_{j}^{*}\left(x_{n}\right) \rightarrow 0$ for every $j$, and hence, $\left\|x_{n}^{c}\right\| \rightarrow 0$. This implies that the restricted semigroup $\left\{\left.G_{t}\right|_{X_{s} \oplus X_{u}}\right\}_{t \geq 0}$ satisfies the same hypotheses (1p),(2h), but is hyperbolic now. By the preceding argument it is not possible.

This contradiction finishes the proof.

Let us turn to the proof of Theorem 1.1.

In papers $[\mathbf{1 8}, \mathbf{2 0}]$ a weakly null sequence of divergence free vector fields $\left\{x_{n}\right\} \subset$ $L_{\text {div }}^{2}\left(T^{3}\right)$ satisfying the conditions of Proposition 2.1 was constructed. We reproduce the main steps of the construction here for convenience of the reader.

Let us fix a Lyapunov-Oseledets exponent $\mu$ of the b-cocycle determined by (1.5). Then there exists a point $\left(x_{0}, \xi_{0}\right) \in \mathbb{K}$ and a vector $b_{0} \in \mathbb{R}^{3}$ such that

$$
\mu=\lim _{t \rightarrow \infty} \frac{1}{t} \log \left|B_{t}\left(x_{0}, \xi_{0}\right) b_{0}\right| \text {. }
$$

It is shown in [18] that every representative of the Euler semigroup, $G_{t}$, is equal, modulo compact perturbation, to the following pseudo-differential operator

$$
P_{t} w(x)=\sum_{q \in \mathbb{Z}^{3} \backslash\{0\}} B_{t}\left(\varphi_{-t}(x), q\right) \hat{w}(q) e^{i q \cdot \varphi_{-t}(x)} .
$$

Let us take a scalar function $h \in L^{2}\left(\mathbb{T}^{3}\right),\|h\|=1$ with Fourier transform supported in the ball of radius $R$, and its weight concentrated around $x_{0}$. Fix a $\delta>0$ and choose $q_{\delta} \in \mathbb{Z}^{3}$ so that $\left|\xi_{0}-\frac{q_{\delta}}{\left|q_{\delta}\right|}\right|<\delta$, and $q_{\delta} / \delta \in \mathbb{Z}^{3}$. We define

$$
\begin{aligned}
w_{\delta} & =\delta \nabla \times\left(\frac{i q_{\delta}}{\left|q_{\delta}\right|^{2}} \times b_{0} \cdot h(y) e^{i \frac{q_{\delta}}{\delta} \cdot y}\right) \\
& =b_{0} h(x) e^{i \frac{q_{\delta}}{\delta} \cdot x}+O(\delta) .
\end{aligned}
$$


So, $\left\|w_{\delta}\right\|=1+O(\delta)$. Furthermore,

$$
\hat{w}_{\delta}(q)=-\delta \cdot q \times\left(\frac{q_{\delta}}{\left|q_{\delta}\right|^{2}} \times b_{0}\right) \hat{h}\left(q-q_{\delta} / \delta\right) .
$$

Using the homogeneity and smoothness of $B_{t}$ in the second variable, we obtain

$$
\begin{aligned}
P_{t} w_{\delta}(x) & =-\sum_{q \in \mathbb{Z}^{3} \backslash\{0\}} B_{t}(x, q) \delta q \times\left(\frac{q_{\delta}}{\left|q_{\delta}\right|^{2}} \times b_{0}\right) \hat{h}\left(q-q_{\delta} / \delta\right) e^{i q \cdot x} \\
& =-e^{i \frac{q_{\delta}}{\delta} \cdot x} \sum_{|q| \leq R} B_{t}\left(x, \delta q+q_{\delta}\right)\left(\delta q+q_{\delta}\right) \times\left(\frac{q_{\delta}}{\left|q_{\delta}\right|^{2}} \times b_{0}\right) \hat{h}(q) e^{i q \cdot x} \\
& =-e^{i \frac{q_{\delta}}{\delta} \cdot x} \sum_{|q| \leq R} B_{t}\left(x, q_{\delta}\right) q_{\delta} \times\left(\frac{q_{\delta}}{\left|q_{\delta}\right|^{2}} \times b_{0}\right) \hat{h}(q) e^{i q \cdot x}+O(\delta) \\
& =e^{i \frac{q_{\delta}}{\delta} \cdot x} \sum_{|q| \leq R} B_{t}\left(x, q_{\delta}\right) b_{0} \hat{h}(q) e^{i q \cdot x}+O(\delta) \\
& =e^{i \frac{q_{\delta}}{\delta} \cdot x} B_{t}\left(x, q_{\delta}\right) b_{0} h(x)+O(\delta) .
\end{aligned}
$$

We can let $R \rightarrow \infty$ as $\delta \rightarrow 0$ slow enough to preserve the limits $O(\delta) \rightarrow 0$ and $w_{\delta} \rightarrow 0$ weakly, and so that $h=h_{\delta}$ eventually concentrates at $x_{0}$ as $\delta \rightarrow 0$. Thus,

$$
\left\|P_{t} w_{\delta}\right\|=\left\|B_{t}\left(\varphi_{-t}(\cdot), q_{\delta}\right) b_{0} h_{\delta}\left(\varphi_{-t}(\cdot)\right)\right\|+O(\delta) \rightarrow\left|B_{t}\left(x_{0}, \xi_{0}\right) b_{0}\right| .
$$

On the other hand,

$$
\left\|G_{t} w_{\delta}-P_{t} w_{\delta}\right\| \rightarrow 0, \text { as } \delta \rightarrow 0
$$

since the difference is compact. So, to conclude the proof we set $x_{n}=w_{1 / n}$ and $f(t)=\left|B_{t}\left(x_{0}, \xi_{0}\right) b_{0}\right|$.

\section{Aperiodic Case}

In this section we outline a scheme of proving Theorem 1.2.

As we mentioned earlier, the presence of high oscillations makes it impossible to single out one particular point $z \in \mathbb{C}$ in the actual spectrum of $L$. Instead, we let $z$ vary on the vertical line $\operatorname{Re} z=\mu$, for some aperiodic Lyapunov-Oseledets exponent $\mu$, and we show that

$$
\left\|(L-\mu-i / \varepsilon)^{-1}\right\| \rightarrow \infty,
$$

as $\varepsilon \rightarrow 0$. Choosing $\varepsilon=(2 \pi k+\zeta)^{-1}$ for $k=1,2, \ldots$ and $\zeta$ arbitrary, one gets under the hypothesis of Gearhart's theorem [12], which implies that $e^{(\mu+i \zeta) t} \in \sigma\left(G_{t}\right)$.

Our basic idea is to construct a sequence of vector fields $\omega_{\varepsilon, N} \in L_{\mathrm{div}}^{2}\left(T^{3}\right)$, $\varepsilon, N>0$, localized about the orbit of point $x_{0}$ given by (1.6), so that

$$
\limsup _{\varepsilon \rightarrow 0}\left\|(L-\mu-i / \varepsilon) \omega_{\varepsilon, N}\right\| /\left\|\omega_{\varepsilon, N}\right\| \leq \alpha(N),
$$

where $\alpha(N)$ is some function not bounded away from zero as $N \rightarrow \infty$. In our construction, parameter $N$ will be naturally restricted to the largest period of an orbit passing near $x_{0}$. So, in order to let $N$ assume large values, we have to have long orbits in any neighborhood of $x_{0}$. This will be achieved by the aperiodicity condition.

So, let us fix an aperiodic Lyapunov-Oseledets exponent $\mu$ with $x_{0}, \xi_{0}$, and $b_{0}$ as in (1.6). For technical reasons, if $\xi_{0} \perp u\left(x_{0}\right)$, then we fix another $\xi_{1}$ close to 
$\xi_{0}$ and not perpendicular to $u\left(x_{0}\right)$. This assumption is not restrictive and will be eliminated later.

Since $\mu$ is aperiodic we can choose $x_{0}$ to be aperiodic. In this case, for any given neighborhood $U$ of $x_{0}$ and $N>0$ we find an $x_{1}$ with period greater than $2 N$. Clearly, if $U$ is sufficiently small, then $\xi_{1} \not \perp u\left(x_{1}\right)$.

Denote by $\Sigma$ a planar tile centered at $x_{1}$ and perpendicular to $\xi_{1}$. Let us take $\Sigma$ of size $\sqrt[4]{\varepsilon} \times \sqrt[4]{\varepsilon}$ for $\varepsilon$ so small that every point in the flow-box $\mathcal{F}_{\varepsilon, N}=\left\{\varphi_{t}(\Sigma)\right\}_{0 \leq t \leq N}$ is uniquely determined by the corresponding $\sigma \in \Sigma$ and $t \in[0, N]$.

Our function $\omega_{\varepsilon, N}$ is defined inside this flow-box $\mathcal{F}_{\varepsilon, N}$ by the following expression

$$
\omega_{\varepsilon, N}=\frac{\varepsilon}{i} \nabla \times\left(\frac{\nabla S \times f}{|\nabla S|^{2}} e^{i S / \varepsilon}\right)
$$

where $S$ is a scalar-valued frequency function, and $f$ is a vector amplitude field supported in the flow-box. Notice that $\omega_{\varepsilon, N}$ being the rotor of a vector field is automatically divergence free.

Let us specify $S$ and $f$.

Since $\nabla S$ plays the role of a wave-vector, we want it to evolve along the orbit according to the $\xi$-equation in (1.5) with initial condition being a multiple of $\xi_{1}$. Setting

$$
S\left(x=\varphi_{t}(\sigma)\right)=t,
$$

for all $x \in \mathcal{F}_{\varepsilon, N}$, we see that $\left.S\right|_{\Sigma} \equiv 0$ and hence, $\left.\nabla S\right|_{\Sigma}$ is indeed proportional to $\xi_{1}$. Moreover, $S\left(\varphi_{t}(x)\right)-S(x)=t$ for all $x \in \mathcal{F}_{\varepsilon, N}$. Taking the gradient at $x=\sigma$ we obtain

$$
\begin{aligned}
\partial \varphi_{t}^{\top}(\sigma) \nabla S\left(\varphi_{t}(\sigma)\right) & =\nabla S(\sigma) \\
\nabla S\left(\varphi_{t}(\sigma)\right) & =\partial \varphi_{t}^{-\top}(\sigma) \nabla S(\sigma) .
\end{aligned}
$$

The last identity expresses the fact that $\nabla S\left(\varphi_{t}(\sigma)\right)$ is a solution to the $\xi$-equation in $t$. Also, directly from the definition we infer

$$
(u, \nabla) S=\frac{d S\left(\varphi_{t}(\sigma)\right)}{d t} \equiv 1 .
$$

For functions $\beta \in C_{0}^{\infty}(\Sigma)$ and $\gamma \in C_{0}^{\infty}(0, N)$ to be specified later, we define the amplitude field by

$$
f\left(x=\varphi_{t}(\sigma)\right)=\beta(\sigma) \gamma(t) e^{-\mu t} b(t) \int_{0}^{t} \frac{\gamma(s)}{e^{-\mu s}|b(s)|} d s
$$

where $\sigma \in \Sigma, 0 \leq t \leq N$, and $b(t)=b\left(t, \sigma, \xi_{1}, b_{0}\right)=B_{t}\left(\sigma, \xi_{1}\right) b_{0}$ is the solution to the $b$-equation (1.5) with initial data $\sigma, \xi_{1}, b_{0}$. Recall that $\sigma \sim x_{0}, \xi_{1} \sim \xi_{0}$ and $b_{0}$ is taken from (1.6).

It will be beneficial to represent $\omega_{\varepsilon, N}$ as a sum of oscillating and negligible terms. Namely, by direct computation,

$$
\omega_{\varepsilon, N}=-f \cdot e^{i S / \varepsilon}+\frac{\varepsilon}{i} e^{i S / \varepsilon} \nabla \times\left(\frac{\nabla S \times f}{|\nabla S|^{2}}\right) .
$$

Denoting $g=f \cdot e^{i S / \varepsilon}$ and $\omega_{0}=\nabla \times\left(\frac{\nabla S \times f}{|\nabla S|^{2}}\right)$ we rewrite

$$
\omega_{\varepsilon, N}=-g+\frac{\varepsilon}{i} e^{i S / \varepsilon} \omega_{0}
$$


Let $z=\mu+i / \varepsilon$ be our spectral parameter. Then, the formula for $g$ reads explicitly

$$
g\left(x=\varphi_{t}(\sigma)\right)=\beta(\sigma) \gamma(t) e^{-z t} b(t) \int_{0}^{t} \frac{\gamma(s)}{e^{-\mu s}|b(s)|} d s .
$$

In the next section we investigate the action of $(L-z)$ on $\omega_{\varepsilon, N}$. In particular, we adjust the functions $\beta$ and $\gamma$ properly to make the energy norm $\left\|(L-z) \omega_{\varepsilon, N}\right\|$ small relative to $\left\|\omega_{\varepsilon, N}\right\|$.

\section{Action of $L$}

Using formulas (3.1), (3.3), and (3.4), after tedious but straightforward computations, we obtain at $x=\varphi_{t}(\sigma)$

$$
\begin{gathered}
(-(u, \nabla)-\partial u) \omega_{\varepsilon, N}=\beta(\sigma) \gamma^{\prime}(t) e^{-z t} b(t) \int_{0}^{t} \frac{\gamma(s)}{e^{-\mu s}|b(s)|} d s-z g \\
+\beta(\sigma) \gamma(t) e^{-z t} b^{\prime}(t) \int_{0}^{t} \frac{\gamma(s)}{e^{-\mu s}|b(s)|} d s+\beta(\sigma) e^{-z t} b(t) \frac{\gamma^{2}(t)}{e^{-\mu t}|b(t)|} \\
+\partial u \cdot g-e^{i S / \varepsilon} \omega_{0}+\frac{\varepsilon}{i} e^{i S / \varepsilon}((u, \nabla)+\partial u) \omega_{0} .
\end{gathered}
$$

Since the Leray projection is orthogonal, we have

$$
\left\|(L-z) \omega_{\varepsilon, N}\right\| \leq\left\|(-(u, \nabla)-\partial u) \omega_{\varepsilon, N}-z \omega_{\varepsilon, N}-\nabla p\right\|,
$$

for any scalar function $p$. Let us choose

$$
p=\frac{\varepsilon}{i} e^{i S / \varepsilon} 2\left(\partial u \cdot f, \frac{\nabla S}{|\nabla S|^{2}}\right)=\frac{\varepsilon}{i} e^{i S / \varepsilon} q .
$$

Then

$$
\nabla p=\nabla S e^{i S / \varepsilon} q+\frac{\varepsilon}{i} e^{i S / \varepsilon} \nabla q .
$$

By construction, $b(t)$ solves the b-equation, i.e.

$$
b^{\prime}=\partial u \cdot b+2\left(\partial u \cdot b, \frac{\nabla S}{|\nabla S|}\right) \frac{\nabla S}{|\nabla S|} .
$$

Combining the last two identities with (4.1), we evaluate at $x=\varphi_{t}(\sigma)$

$$
\begin{gathered}
(-(u, \nabla)-\partial u) \omega_{\varepsilon, N}-z \omega_{\varepsilon, N}-\nabla p=\beta(\sigma) \gamma^{\prime}(t) e^{-z t} b(t) \int_{0}^{t} \frac{\gamma(s)}{e^{-\mu s}|b(s)|} d s \\
+\beta(\sigma) e^{-z t} b(t) \frac{\gamma^{2}(t)}{e^{-\mu t}|b(t)|}-\frac{\varepsilon}{i} e^{i S / \varepsilon}\left((u, \nabla) \omega_{0}-\nabla q+\partial u \cdot \omega_{0}\right) .
\end{gathered}
$$

Suppressing variables we get the following inequality,

$$
\begin{gathered}
\left\|(L-z) \omega_{\varepsilon, N}\right\| \leq\left\|\beta \gamma^{\prime} e^{-\mu t} b \int_{0}^{t}\right\|+\left\|\beta \gamma^{2}\right\|+\varepsilon\left\|(u, \nabla) \omega_{0}-\nabla q+\partial u \cdot \omega_{0}\right\| \\
=I_{1}+I_{2}+\varepsilon I_{3} .
\end{gathered}
$$

Our next goal is to make the right hand side infinitely small in comparison with the norm of $\omega_{\varepsilon, N}$ as $\varepsilon \rightarrow 0$ and $N \rightarrow \infty$. We achieve it by a proper choice of functions $\beta$ and $\gamma$, which are still at our disposal. Their definition would be more transparent if to work with $\sigma$ and $t$ rather than $x$ variable. To this end, let us introduce suitable transformation of coordinates and change variables in the integrals $I_{1}, I_{2}$. 
We define a map from $\mathcal{S}=[0, N] \times \Sigma$ onto $\mathcal{F}_{\varepsilon, N}$ by

$$
H(t, \sigma)=\varphi_{t}(\sigma) .
$$

A direct computation shows that

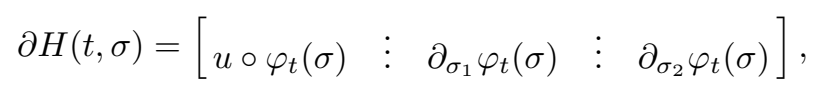

where $\sigma=\left(\sigma_{1}, \sigma_{2}\right)$. Let $e_{k}$ be the unit vector in the $\sigma_{k}$-th direction. Then

$$
\partial H(t, \sigma)=\partial \varphi_{t}(\sigma)\left[u(\sigma) \quad \vdots \quad e_{1} \quad \vdots \quad e_{2}\right] .
$$

Consequently, the quantity

$$
|\operatorname{det} \partial H(t, \sigma)|=\left|\left(u(\sigma), e_{1} \times e_{2}\right)\right|,
$$

denoted $d(\sigma)$ in the sequel, does not depend on $t$. Besides, $d(0) \neq 0$ due to our assumption that $\xi_{1} \not \perp u\left(x_{1}\right)$.

So, making the change of variables in $I_{1}$ and $I_{2}$ we get

$$
\begin{aligned}
I_{1}^{2} & =\int_{\mathcal{S}} d(\sigma) \beta^{2}(\sigma)\left|e^{-\mu t} b(t) \gamma^{\prime}(t) \int_{0}^{t} \frac{\gamma(s)}{e^{-\mu s}|b(s)|} d s\right|^{2} d t d \sigma \\
I_{2}^{2} & =\int_{\mathcal{S}} d(\sigma) \beta^{2}(\sigma) \gamma^{4}(t) d t d \sigma .
\end{aligned}
$$

Let us choose $\beta \in L^{2}(\Sigma, d \sigma)$ so that $\|\beta\|_{L^{2}(\Sigma, d \sigma)}=1$ and

$$
\left\|\partial^{(\alpha)} \beta\right\|_{L^{2}(\Sigma, d \sigma)} \leq 1 / \sqrt{\varepsilon}
$$

for $|\alpha| \leq 2$. The latter is possible due to our assumption on the size of $\Sigma$. Since all the functions involved in $I_{3}$ contain at most the second derivative of $\beta$, we immediately obtain $\varepsilon I_{3} \rightarrow 0$ as $\varepsilon \rightarrow 0$. Also,

$$
\begin{array}{ll}
I_{1}^{2} \rightarrow d(0) \int_{0}^{N}\left|e^{-\mu t} b(t) \gamma^{\prime}(t) \int_{0}^{t} \frac{\gamma(s)}{e^{-\mu s}|b(s)|} d s\right|^{2} d t & \stackrel{\text { def }}{=} d(0) J_{1}(N) \\
I_{2}^{2} \rightarrow d(0) \int_{0}^{N} \gamma^{4}(t) d t & \stackrel{\text { def }}{=} d(0) J_{2}(N) .
\end{array}
$$

As to the functions $\omega_{\varepsilon, N}$, using representation (3.3) and performing similar estimates, we obtain

$$
\left\|\omega_{\varepsilon, N}\right\|^{2} \rightarrow d(0) \int_{0}^{N}\left|e^{-\mu t} b(t) \gamma(t) \int_{0}^{t} \frac{\gamma(s)}{e^{-\mu s}|b(s)|} d s\right|^{2} d t \stackrel{\text { def }}{=} d(0) J(N) .
$$

We now define $\gamma$ as follows,

$$
\gamma(t)= \begin{cases}0, & t \leq 0 \\ t / \sqrt{N}, & 0<t \leq 1 \\ 1 / \sqrt{N}, & 1<t \leq N-1 \\ (N-t) / \sqrt{N}, & N-1<t \leq N\end{cases}
$$

smoothed out at $0,1, N-1, N$.

This implies that $J_{2}(N) \sim 1 / N$, where $A \sim B$ means there is a constant $c$ such that $c^{-1} A<B<c A$. 
To estimate $J_{1}$ and $J$ first notice that $b(t) \sim b(\tau)$ whenever $|t-\tau|<1$. So, denoting $h(t)=h\left(t, x_{1}, \xi_{1}, b_{0}\right)=e^{-\mu t}\left|b\left(t, x_{1}, \xi_{1}, b_{0}\right)\right|$ we have

$$
\begin{aligned}
J_{1}(N) & \sim \frac{1}{N^{2}}\left[\int_{0}^{N} \frac{h(N)}{h(t)} d t\right]^{2} \\
J(N) & \sim \frac{1}{N^{2}} \int_{0}^{N}\left[\int_{0}^{t} \frac{h(t)}{h(s)} d s\right]^{2} d t .
\end{aligned}
$$

Combining the obtained estimates we see that

$$
\begin{aligned}
\limsup _{\varepsilon \rightarrow 0}\left\|(L-z) \omega_{\varepsilon, N}\right\|^{2} /\left\|\omega_{\varepsilon, N}\right\|^{2} & \lesssim \frac{d(0) J_{1}(N)+d(0) J_{2}(N)}{d(0) J(N)} \\
& \sim \frac{\frac{1}{N}\left[\int_{0}^{N} \frac{h(N)}{h(t)} d t\right]^{2}+1}{\frac{1}{N} \int_{0}^{N}\left[\int_{0}^{t} \frac{h(t)}{h(s)} d s\right]^{2} d t}=\frac{S_{1}(N)+1}{S(N)}=\alpha(N) .
\end{aligned}
$$

Hence,

$$
\liminf _{\varepsilon \rightarrow 0}\left\|(L-z)^{-1}\right\| \gtrsim \alpha^{-1 / 2}(N) .
$$

By the construction, $\alpha(N)$ depends only on $x_{1}, \xi_{1}, b_{0}$ but not on the angle between $\xi_{1}$ and $u\left(x_{1}\right)$. So we can let $\xi_{1} \rightarrow \xi_{0}$ in (4.2) preserving the inequality. Furthermore, due to our aperiodicity assumption, the point $x_{1}$ can be chosen as close to $x_{0}$ as we wish. Hence, we can let $x_{1} \rightarrow x_{0}$ too. As a result, inequality (4.2) remains valid for all $N>0$ and for $x_{0}, \xi_{0}, b_{0}$ originally given by (1.6). In particular, the function $h$ in the formula for $\alpha$ has Lyapunov index 0, i.e

$$
\lim _{t \rightarrow \infty} \frac{1}{t} \log h(t)=0
$$

So, it remains to prove that $\alpha(N)$ is not bounded away from zero as $N \rightarrow \infty$.

\section{Functions with Zero Lyapunov Index}

Let us rewrite $\alpha(N)$ as the sum

$$
\alpha(N)=\frac{S_{1}(N)}{S(N)}+\frac{1}{S(N)} .
$$

Clearly, it suffices to show that $S_{1}(N) / S(N)$ is not bounded away from zero and $S(N) \rightarrow \infty$ as $N \rightarrow \infty$.

The proof of the first assertion is based on the Grönwall Lemma, while the second one requires a more delicate probabilistic argument.

Lemma 5.1. Let $h: \mathbb{R} \rightarrow \mathbb{R}^{+}$be any function of zero Lyapunov index and let

$$
\begin{aligned}
S_{1}(N) & =\frac{1}{N} \int_{0}^{N}\left[\int_{0}^{t} \frac{h(t)}{h(s)} d s\right]^{2} d t, \\
S(N) & =\frac{1}{N}\left[\int_{0}^{N} \frac{h(N)}{h(t)} d t\right]^{2} .
\end{aligned}
$$

Then $S_{1}(N) / S(N)$ is not bounded away from zero as $N \rightarrow \infty$. 
Proof. Assume, on the contrary, that there is a constant $c>0$ such that $S_{1}(N) \geq c S(N)$. Denoting

$$
f(t)=\left[\int_{0}^{t} \frac{h(t)}{h(s)} d s\right]^{2}
$$

we can rewrite the inequality above as

$$
f(N) \geq c \int_{0}^{N} f(t) d t=c_{1}+c \int_{1}^{N} f(t) d t .
$$

So, by the Grönwall Lemma

$$
f(N) \gtrsim e^{c N}
$$

which, possibly with different $c$, implies

$$
\frac{1}{N} \int_{0}^{N} \frac{h(N)}{h(s)} d s \gtrsim \frac{1}{N} e^{c N} .
$$

Thus, there is an $s \in[0, N]$ such that

$$
h(N) \gtrsim h(s) \frac{1}{N} e^{c N} .
$$

Since $h$ has Lyapunov index zero, we can write

$$
h(s) \gtrsim e^{-c s / 2} .
$$

Hence,

which leads to a contradiction.

$$
h(N) \gtrsim \frac{1}{N} e^{c N / 2},
$$

For the second assertion, it is more convenient to rewrite $S(N)$ in an equivalent discrete form. Namely,

$$
S(N) \sim \frac{1}{N} \sum_{n=1}^{N}\left[\sum_{k=1}^{n} \frac{h(n)}{h(k)}\right]^{2} .
$$

This readily follows from the fact that $h(t) \sim h(\tau)$, whenever $|t-\tau|<1$.

LEMma 5.2. Let $h: \mathbb{N} \rightarrow \mathbb{R}^{+}$be any function of zero Lyapunov index. Then

$$
\lim _{N \rightarrow \infty} \frac{1}{N} \sum_{n=1}^{N}\left[\sum_{k=1}^{n} \frac{h(n)}{h(k)}\right]^{2}=\infty
$$

Proof. Let us fix an arbitrary $M \in \mathbb{N}$ and introduce the following sets

$$
\begin{aligned}
P_{n} & =\left\{k \leq n: \frac{h(n)}{h(k)}>1 / 2\right\} \\
Q_{N} & =\left\{n \leq N:\left|P_{n}\right|>M\right\}
\end{aligned}
$$

It suffices to prove that

$$
\liminf _{N \rightarrow \infty}\left|Q_{N}\right| / N \geq 1 / 8
$$


Indeed, then for sufficiently large $N$ we have

$$
\begin{aligned}
\frac{1}{N} \sum_{n=1}^{N}\left[\sum_{k=1}^{n} \frac{h(n)}{h(k)}\right]^{2} & \geq \frac{1}{N} \sum_{n \in Q_{N}}\left[\sum_{k \in P_{n}} \frac{h(n)}{h(k)}\right]^{2} \\
& \geq \frac{1}{N} \sum_{n \in Q_{N}}\left(\left|P_{n}\right| / 2\right)^{2} \\
& \geq \frac{1}{N}\left|Q_{N}\right| M^{2} / 4 \geq \frac{1}{N} \frac{N}{40} M^{2}=M^{2} / 40 .
\end{aligned}
$$

In order to prove (5.1) we argue as follows.

Suppose (5.1) fails. Then we can find an arbitrarily large $N$ such that $\left|Q_{N}\right|<$ $N / 8$ or, taking the complement in $[0, N],\left|Q_{N}^{c}\right| \geq 7 N / 8$. Let as fix one such $N$.

Obviously, there exists an $n_{1} \in Q_{N}^{c}$ such that $n_{1} \geq 7 N / 8$. Hence, $\left|P_{n_{1}}\right| \leq M$.

We continue by selecting the first integer $n_{2}$ to the left of $n_{1}$ that belongs to $P_{n_{1}}^{c} \cap Q_{N}^{c}$. Then $\left|P_{n_{2}}\right| \leq M,\left(n_{2}, n_{1}\right) \cap P_{n_{1}}^{c} \cap Q_{N}^{c}=\emptyset$ and

$$
h\left(n_{1}\right) \leq \frac{1}{2} h\left(n_{2}\right) .
$$

Similarly, we find $n_{3}<n_{2}$ the first in $P_{n_{2}}^{c} \cap Q_{N}^{c}$. Then $\left|P_{n_{3}}\right| \leq M$ and

$$
h\left(n_{2}\right) \leq \frac{1}{2} h\left(n_{3}\right) .
$$

On the k-th step, we take $n_{k}<n_{k-1}$ to be the first in $P_{n_{k-1}}^{c} \cap Q_{N}^{c}$ so that $\left|P_{n_{k}}\right| \leq M$ and

$$
h\left(n_{k-1}\right) \leq \frac{1}{2} h\left(n_{k}\right)
$$

The process will continue at least until

$$
n_{k} \leq N / 8+M,
$$

for otherwise $\left|P_{n_{k}}^{c}\right|>n_{k}-M>N / 8$ and $\left|Q_{N}^{c}\right| \geq 7 N / 8$, so $P_{n_{k}}^{c} \cap Q_{N}^{c} \neq \emptyset$. Let $k$ be the first integer satisfying (5.2).

Then,

$$
h\left(n_{1}\right) \leq \frac{1}{2^{k-1}} h\left(n_{k}\right) .
$$

On the other hand, by our construction,

$$
\begin{aligned}
& \left(n_{i+1}, n_{i}\right) \cap P_{n_{i}}^{c} \cap Q_{N}^{c}=\emptyset \\
& \left(n_{i+1}, n_{i}\right)=\left(P_{n_{i}} \cup Q_{N}\right) \cap\left(n_{i+1}, n_{i}\right) .
\end{aligned}
$$

So,

$$
n_{i}-n_{i+1} \leq M+\left|Q_{N} \cap\left(n_{i+1}, n_{i}\right)\right| .
$$

Consequently,

$$
\begin{aligned}
7 N / 8-N / 8-M \leq n_{1}-n_{k} & =\sum_{i=1}^{k-1} n_{i}-n_{i+1} \\
& \leq(k-1) M+\sum_{i=1}^{k-1}\left|Q_{N} \cap\left(n_{i+1}, n_{i}\right)\right| \\
& \leq(k-1) M+\left|Q_{N}\right|<(k-1) M+N / 8 .
\end{aligned}
$$


From this we get the necessary estimate on $k$ :

$$
k \geq \frac{5 N}{8 M} \text {. }
$$

Using (5.3) we obtain

$$
\begin{aligned}
\frac{1}{n_{1}} \log \left[\frac{h\left(n_{1}\right)}{h\left(n_{k}\right)}\right] \leq & \frac{k-1}{n_{1}} \log (1 / 2) \leq\left(\frac{5 N}{8 M}-1\right) \frac{8}{7 N} \log (1 / 2) \\
& =\left(\frac{5}{7 M}-\frac{8}{7 N}\right) \log (1 / 2)
\end{aligned}
$$

Passing to the limit as $N \rightarrow \infty$ shows that the Lyapunov index of $h$ is negative, which contradicts our hypothesis.

\section{References}

[1] L. Belenkaya, S. Friedlander, and V. Yudovich, The unstable spectrum of oscillating shear flows, SIAM J. Appl. Math. 59 (1999), no. 5, 1701-1715 (electronic). MR 2000e:76051

[2] Felix E. Browder, On the spectral theory of elliptic differential operators. I, Math. Ann. 142 (1960/1961), 22-130. MR 35 \#804

[3] S. Chandrasekhar, Hydrodynamic and hydromagnetic stability, The International Series of Monographs on Physics, Clarendon Press, Oxford, 1961. MR 23 \#B1270

[4] Carmen Chicone and Yuri Latushkin, Evolution semigroups in dynamical systems and differential equations, American Mathematical Society, Providence, RI, 1999. MR 2001e:47068

[5] Rafael de la Llave, Hyperbolic dynamical systems and generation of magnetic fields by perfectly conducting fluids, Geophys. Astrophys. Fluid Dynam. 73 (1993), no. 1-4, 123-131, Magnetohydrodynamic stability and dynamos (Chicago, IL, 1992). MR 95k:58124b

[6] P. G. Drazin and W. H. Reid, Hydrodynamic stability, Cambridge Monographs on Mechanics and Applied Mathematics, Cambridge University Press, Cambridge, 1982. MR 84a:76001

[7] Klaus-Jochen Engel and Rainer Nagel, One-parameter semigroups for linear evolution equations, Springer-Verlag, New York, 2000, With contributions by S. Brendle, M. Campiti, T. Hahn, G. Metafune, G. Nickel, D. Pallara, C. Perazzoli, A. Rhandi, S. Romanelli and R. Schnaubelt. MR 2000i: 47075

[8] Susan Friedlander, Lectures on stability and instability of an ideal fluid, Hyperbolic equations and frequency interactions (Park City, UT, 1995), IAS/Park City Math. Ser., vol. 5, Amer. Math. Soc., Providence, RI, 1999, pp. 227-304. MR 2000a:76001

[9] Susan Friedlander, Walter Strauss, and Misha Vishik, Nonlinear instability in an ideal fluid, Ann. Inst. H. Poincaré Anal. Non Linéaire 14 (1997), no. 2, 187-209. MR 99a:76057

[10] Susan Friedlander, Misha Vishik, and Victor Yudovich, Unstable eigenvalues associated with inviscid fluid flows, J. Math. Fluid Mech. 2 (2000), no. 4, 365-380. MR 2001m:76009

[11] Susan Friedlander and Misha M. Vishik, Instability criteria in fluid dynamics, Topological aspects of the dynamics of fluids and plasmas (Santa Barbara, CA, 1991), Kluwer Acad. Publ., Dordrecht, 1992, pp. 535-549. MR 94d:76038

[12] Larry Gearhart, Spectral theory for contraction semigroups on Hilbert space, Trans. Amer. Math. Soc. 236 (1978), 385-394. MR 57 \#1191

[13] Horace Lamb, Hydrodynamics, sixth ed., Cambridge Mathematical Library, Cambridge University Press, Cambridge, 1993, With a foreword by R. A. Caflisch [Russel E. Caflisch]. MR 96f: 76001

[14] Yuri Latushkin and Misha Vishik, Linear stability in an ideal incompressible fluid, Commun. Math. phys. 233 (2003), 439-461.

[15] Zhiwu Lin, Instability of some ideal plane flows, (preprint).

[16] V. I. Oseledec, A multiplicative ergodic theorem. Characteristic Ljapunov, exponents of dynamical systems, Trudy Moskov. Mat. Obšč. 19 (1968), 179-210. MR 39 \#1629

[17] Lord Rayleigh, On the stability or instability of certain fluid motions, Proc. Lond. Math. Soc. 9 (1880), 57-70.

[18] Roman Shvydkoy, A new proof of instability criterion for steady flows of an ideal fluid, (preprint). 
[19] Roman Shvydkoy and Yuri Latushkin, The essential spectrum of the linearized 2D Euler operator is a vertical band, Contemp. Math. 327 (2003), 299 - 304.

[20] Misha Vishik, Spectrum of small oscillations of an ideal fluid and Lyapunov exponents, J. Math. Pures Appl. (9) 75 (1996), no. 6, 531-557. MR 97k:35203

[21] Misha M. Vishik and Susan Friedlander, Dynamo theory methods for hydrodynamic stability, J. Math. Pures Appl. (9) 72 (1993), no. 2, 145-180. MR 95a:76036

Department of Mathematics, 1 University Station C1200, University of Texas, Austin, TX 78712

E-mail address: shvydkoy@math.uic.edu, vishik@math.utexas.edu 